\title{
Serum Lipid Profiles of Ovariectomized Rats Following Short-Term Administration of Cocoa Powder and Ethanolic Extract
}

\author{
Ariza Budi Tunjung Sari ${ }^{\left.{ }^{*}\right)}$, Misnawi ${ }^{1)}$, Pratiwi Pudjiastuti ${ }^{2)}$, and Afaf Baktir ${ }^{2)}$ \\ ${ }^{1)}$ Post-Harvest Laboratory, Indonesian Coffee and Cocoa Research Institute, Jember, Indonesia \\ ${ }^{2}$ Chemistry Department, Faculty of Science and Technology, Airlangga University, Surabaya, Indonesia \\ *)Corresponding author: ariza.bts@gmail.com \\ Received: 11 October 2018 / Accepted: 28 May 2019
}

\begin{abstract}
Estrogen depletion increases the risk of dyslipidemia by triggering higher levels of total cholesterol (TC), triglyceride (TG), and low density lipoprotein (LDL) besides depressed level of high density lipoprotein (HDL). This study was conducted to investigate the potential of cocoa products to affect serum lipid profile in the estrogen-deficient rats. Thirty adult female wistar rats were divided into five groups i.e. four groups contained ovariectomized rats, and one group consisted of intact rats. The test articles were all dissolved in olive oil and administered orally, comprising of $1 \mathrm{~g} / \mathrm{kg}$ body weight (BW) cocoa powder, $1 \mathrm{~g} / \mathrm{kg} \mathrm{BW}$ cocoa extract, $1 \mathrm{mg} / \mathrm{kg} \mathrm{BW}$ estradiol valerate, $10 \mathrm{ml} / \mathrm{kg} \mathrm{BW}$ olive oil as carrier. Intact group was given drinking water. After three-day administration, the rats were terminated and serum lipid profile was observed. The trial obtained ethical approval from the Animal Care and Use Committee, Veterinary Faculty, Airlangga University (Certificate No. 620-KE). The result showed that carrier group developed higher LDL and lower HDL levels, as well as greater LDL/HDL ratio compared to that of intact group. Estradiol valerate group had significantly elevated TG level. Cocoa powder and cocoa extract groups showed small and non-significant changes in TC, TG and HDL. Surprisingly, consumption of cocoa extract that is rich in polyphenols had resulted highest mean of LDL levels among other groups. It was hypothesized that polyphenol in cocoa extract had affected expression of LDL receptors (LDLR) due to an antagonistic activity against estrogen receptor alpha (ERá). To conclude, neither cocoa powder nor cocoa extract exhibits significant estrogenic effect on the serum lipid profile of estrogen-deficient rats.
\end{abstract}

Keywords: Cocoa, estrogen, cholesterol, ovariectomy, polyphenols, triglyceride, low density lipoprotein, high density lipoprotein

\section{INTRODUCTION}

Estrogen deficiency alters serum lipid profile in menopausal women. The change is marked with higher low density lipoprotein (LDL) levels which increases the risk of atherosclerosis (Anagnostis et al., 2015). Estrogen replacement therapy has shown some evidences in suppressing LDL levels thus it is considered having cardioprotective benefit (Escalante et al., 2017).
Interestingly, some of estrogen replacement substances come from polyphenol classes. Extensive studies have revealed estrogenic properties of some polyphenols such as on genistein in soybean, 8-prenylnaringenin in hop seed, and resveratrol in grape (Michel et al., 2013). Polyphenol is abundantly present in cocoa bean. The cocoa polyphenol had shown cardioprotective property by suppressing inflammation and enhancing elasticity of the vascular wall (Baba et al., 2007). 
Nevertheless, the effect of cocoa polyphenol on serum lipid profile during estrogen deficiency is less investigated.

In this study, cocoa was investigated for its ability to substitute estrogen effect on serum lipid profile. For this purpose, female rats were used and the production of endogenous estrogen was stopped by means of ovariectomy which removes both ovaries as the main sources of estrogen hormone. The cocoa powder and extract were used as the test articles to respectively represent a low polyphenol and high polyphenol intake. Following three-day administration, the serum was analyzed for total cholesterol (TC), triglyceride (TG), LDL, and high density lipoprotein (HDL) levels.

\section{MATERIALS AND METHODS}

Two products of cocoa were used in the study which were cocoa powder and cocoa extract. Cocoa powder was prepared from dried cocoa beans that were roasted, cleaned from shells, and separated from the fat. Dried cocoa beans were from forastero variety, collected from farmers around Jember region. Cocoa extract was prepared from fresh beans that were dried, cleaned from shells, separated from the fat and macerated in ethanol. The liquid extract was spray-dried to result powdered extract. The positive control was using estradiol valerate (Progynova, Bayer) which is a synthetical estrogen substitute. All test articles were dissolved in olive oil as carrier, at concentration $0.1 \mathrm{~g} / \mathrm{mL}$.

Thirty wistar rats aged eight weeks old were acclimatized for at least one week and were distributed into five groups. Four groups consisted of ovariectomized rats, each group was constituted of 6 individuals. The procedure for ovariectomy was initiated with anesthesia done through intramuscular injection of $0.1 \mathrm{~mL}$ ketamine and $0.1 \mathrm{~mL}$ xylazine per individual (BW 60-80 g). After removal of both ovaries, rats were allowed to recover for two weeks prior to treatment. One group of six intact rats was prepared as control that represented normal estrogen condition. To this group, the similar procedure was performed but no tissue was removed. During the trial period, rats were given free access towards soy-free feed and water.

The ovariectomized rats received test articles dissolved in olive oil. Test articles consisted of cocoa powder $1 \mathrm{~g} / \mathrm{kg} \mathrm{BW}$, cocoa extract $1 \mathrm{~g} / \mathrm{kg} \mathrm{BW}$, olive oil as carrier $1 \mathrm{~mL} /$ $\mathrm{kg} \mathrm{BW}$, or estradiol valerate $1 \mathrm{mg} / \mathrm{kg} \mathrm{BW}$. The intact rats received water $1 \mathrm{~mL} / \mathrm{kg} \mathrm{BW}$. Administration of test articles was through oral route, once daily for three days. On the fourth day, rats were terminated then the blood samples were collected. The analysis of serum lipid comprised of TC, TG, HDL, and LDL levels measurement done by using spectrophotometer. The atherogenic risk predictors were calculated from the ratio of HDL on TC, LDL on HDL, and log of (TG/ HDL) (Adejor et al., 2017).

Data from each group were the mean from six replications. The data were tested for normality distribution using Saphiro-Wilk test, and transformed into $\log 10$. The analysis of covariance was performed at level of confidence $95 \%(\alpha=0.05)$. Body weight was included as the covariable.

\section{RESULTS AND DISCUSSION}

There were no significant changes in the serum lipid profile in the two weeks postsurgery. This is shown in the carrier group and in the intact group (Figure 1). However, carrier group had slightly lower mean HDL, and higher TC, TG, and LDL levels than intact group. Estrogen substitution with estradiol valerate for three days on the ovariectomized rats resulted no difference in the TC, HDL and LDL levels, compared to two 
former groups. The LDL level tended to decline but the change did not reach statistical significance. TG level in the estradiol valerate group was the highest among other groups.

Cocoa powder group and cocoa extract group obtained some changes which were not significantly different from the carrier group. Both groups showed lower TG, along with elevated TC and HDL levels. LDL levels in the cocoa extract group were significantly higher than any other groups.

The atherogenic risk predictors comprising of HDL/TC, LDL/HDL, and Log (TG/HDL) (Figure 2) showed that all ovariectomized rats developed higher atherogenic risk. These are particularly shown from significantly higher LDL/ HDL ratio $(\mathrm{P}<0.05)$ than intact group.
This finding confirms the cardiprotective property carried out by estrogen.

Slight differences in the lipid profile of the carrier group as compared to intact group showed that the changes take certain time after ovariectomy procedure. However, the carrier group had higher LDL/HDL ratio, which implies dyslipidemia was progressing. It was reported that ovariectomized rats developed dyslipidemia after 12 weeks postsurgery (Saengsirisuwan et al., 2009). Similarly, a very short-term administration of estrogen substitute may not be sufficient to improve serum lipid profile. Therefore, small changes among groups were not surprising within the time frame given in this trial.

The elevated TG level during the use of estradiol valerate was also reported in other study (Mvondo et al., 2011). Oral administration $\mathrm{mg} / \mathrm{dL}$

TC

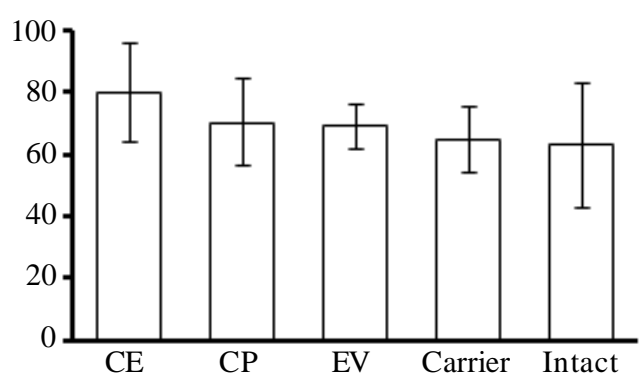

$\mathrm{mg} / \mathrm{dL}$

HDL

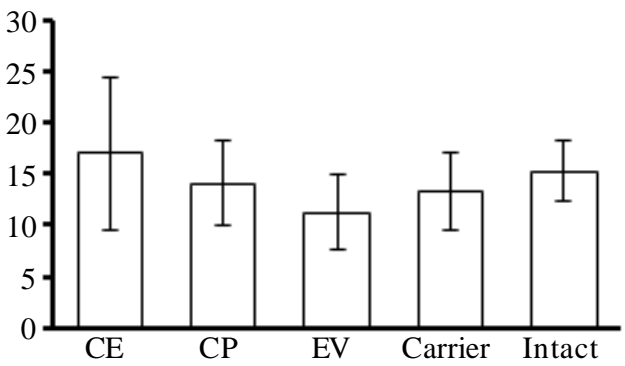

$\mathrm{mg} / \mathrm{dL}$

TG

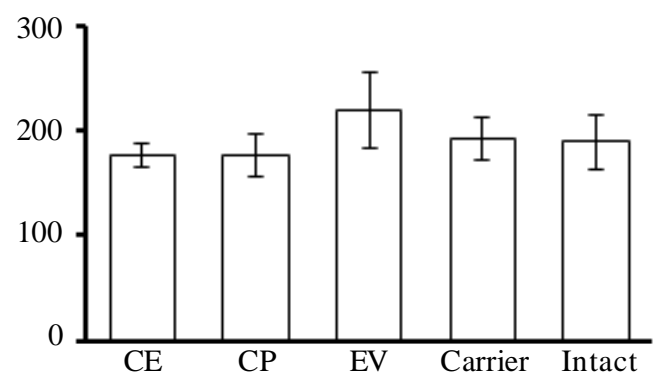

$\mathrm{mg} / \mathrm{dL}$

LDL

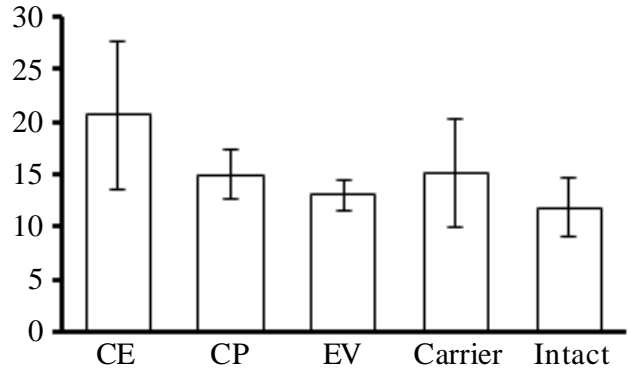

Figure 1. Serum lipid profile of ovariectomized rats given cocoa powder (CP), cocoa extract (CE), estradiol valerate (EV) and olive oil (carrier), compared with intact rats given drinking water (Intact). The bar showed mean of total cholesterol (TC), triglyceride (TG), high density lipoprotein (HDL), and low density lipoprotein (LDL) levels of each group. 


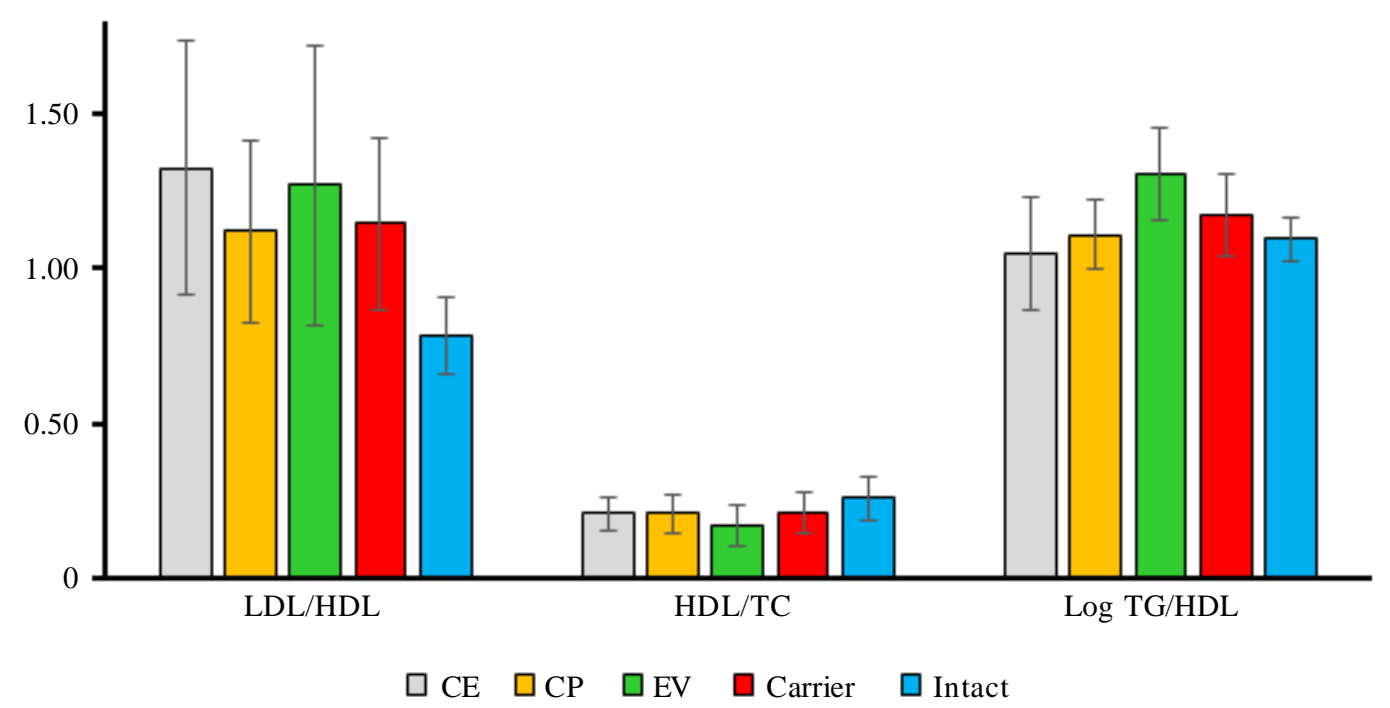

Figure 2. Atherogenic risk predictors in the ovariectomized rats given cocoa powder (CP), cocoa extract $(\mathrm{CE})$, estradiol valerate $(\mathrm{EV})$ and olive oil (carrier), compared with intact rats given drinking water (Intact). The bars show mean values of HDL/LDL, HDL/TC, and Log (TG/HDL) of each group

of estrogen therapy increased TG level, while the transdermal administration did not (Crook et al., 1992; Godsland, 2001). Exogenous estrogen has been reported to decrease activity of hepatic lipase which is responsible for TG clearance from blood, thus serum TG levels remained high (Godsland, 2001; Tikkanen et al., 1979; Urabe et al., 1996).

Estrogen maintains circulating LDL level by upregulating the expression of LDL receptor (LDLR) whose one of its functions is clearing LDL molecules from blood (Ohlsson et al., 2000). In a cell based study, Smith et al. (2004) reported that LDLR mRNA was suppressed in the absence of estrogen, and abundantly present after treatment with $17 \alpha$-ethinyl estradiol, while diminishing dramatically following exposure of antiestrogen ICI 182,780. Its regulation specifically involves estrogen receptor alpha $(\mathrm{ER} \alpha)$, of which one of the mechanisms is through the interaction with steroid receptor binding protein. It has been reported in several studies that diarylpropionitrile, a specific ER $\beta$ agonist, failed to suppress LDL level elevation in estrogen-deficient animals (Xu et al., 2016).

Cocoa is a great source of polyphenols, mainly from a class of flavonoid. It has been attributed with cardioprotective property. The mechanisms are through improvement of cardiovascular elasticity and inhibition of LDL oxidation (Keen et al., 2005). Some studies revealed that consumption of cocoa products also decreased blood LDL levels, but this was found in healthy individuals after long term application (Jia et al., 2010). There is little information on effect of cocoa consumption in relation with estrogen status, such as in perimenopausal and menopausal phases. In this study using estrogen-deficient rats, a significant rise in the LDL levels was observed in the cocoa extract group (Figure 1). The levels are the highest among all ovariectomized groups, which indicated severe LDLR obstruction. Apart from its antioxidative and cardioprotective properties, the polyphenolrich cocoa extract may exhibit antagonistic activity towards ER $\alpha$. 
Polyphenol in the cocoa extract may have also affected thyroid hormone levels. Thyroid hormones reduce LDL level, not by increasing expression of LDLR but by suppressing production of apolipoprotein $\mathrm{B}$ (Goldberg et al., 2012). There have been studies on the inhibitory activity of polyphenols against triiodothyronine (T3) and thyroxine (T4) productions. For instance, green tea polyphenols was reported to inhibit thyroid peroxidase and thyroidal 5'-deiodinase I, the enzymes that induce synthesis of both T3 and T4 (Chandra \& De, 2013).

Thyroid and estrogen signaling both have genomic and non-genomic pathways. The genomic pathway involves nuclear receptors, while non-genomic one interacts with membrane receptors. The non-genomic pathway of thyroid and estrogen hormones is overlapping. When thyroid hormone binds to its membrane receptor, signal transduction is induced. The intermediate proteins playing role in signal transduction, for instance mitogen activated protein kinase (MAPK), are then able to activate ER $\alpha$ (Davis et al., 2011) which is responsible for triggering expression of LDLR (Li et al., 2001). In the case of high serum LDL levels in the cocoa extract group, it is hypothesized that polyphenol in the cocoa extract had decreased thyroid hormones level. This event along with the lack of estrogen following ovariectomized, suppresses activation of ER $\alpha$ and consequently LDLR expression, leading to high LDL level in serum. Future study should investigate the influence of cocoa polyphenol towards ERá via thyroid hormone pathway.

\section{CONCLUSIONS}

Cocoa powder and cocoa extract failed to substitute estrogen in improving serum lipid profile of estrogen deficient rats. Ovariectomized rats consuming both test articles did not experience significant increase in $\mathrm{HDL}$ nor decrease in TG and TC, as compared with ovariectomized rats consuming olive oil as carrier. Cocoa extract group developed high LDL level after treatment, suggesting antagonistic activity against $\mathrm{ER} \alpha$.

\section{ACKNOWLEDGEMENT}

The research was funded by Indonesian Coffee and Cocoa Research Institute. We thank the Biology and Dental Health Departments of Jember University for the surgery facilities. The trial has obtained ethical approval from Animal Care and Use Committee of Veterinary Faculty, Airlangga University (Certificate No. 620-KE).

\section{REFERENCES}

Adejor, E.B.; D.A. Ameh; D.B. James; O.A. Owolabi \& U.S. Ndidi (2017). Effects of Garcinia kola biflavonoid fractions on serum lipid profile and kidney function parameters in hyperlipidemic rats. Clinical Phytoscience, 2, 19.

Anagnostis, P.; J.C. Stevenson; D. Crook; D.G. Johnston \& I.F. Godsland (2015). Effects of menopause, gender and age on lipids and high-density lipoprotein cholesterol subfractions. Maturitas, 81, 62-68.

Baba, S.; N. Osakabe; Y. Kato; M. Natsume; A. Yasuda; T. Kido; K. Fukuda; Y. Muto $\&$ K. Kondo (2007). Continuous intake of polyphenolic compounds containing cocoa powder reduces LDL oxidative susceptibility and has beneficial effects on plasma HDL-cholesterol concentrations in humans. The American Journal of Clinical Nutrition, 85, 709-717.

Chandra, A.K. \& N. De (2013). Catechin induced modulation in the activities of thyroid hormone synthesizing enzymes leading to hypothyroidism. Molecular and Cellular Biochemistry, 374, 37-48.

Crook, D.; M.P.Cust; K.F. Gangar; M. Worthington; T.C. Hillard; J.C. Stevenson; M.I. White- 
head \& V. Wynn (1992). Comparison of transdermal and oral estrogenprogestin replacement therapy: Effects on serum lipids and lipoproteins. American Journal of Obstetrics and Gynecology, 166, 950-955.

Davis, P.J.; H.-Y. Lin; S.A. Mousa; M.K. Luidens; A.A. Hercbergs; M. Wehling \& F.B. Davis (2011). Overlapping nongenomic and genomic actions of thyroid hormone and steroids. Steroids, 76, 829-833.

Escalante, C.G.; S.Q. Mora \& L.N. Bolaños (2017). Hormone replacement therapy reduces lipid oxidation directly at the arterial wall: A possible link to estrogens' cardioprotective effect through atherosclerosis prevention. Journal of Mid-life Health, 8, 11.

Godsland, I.F. (2001). Effects of postmenopausal hormone replacement therapy on lipid, lipoprotein, and apolipoprotein (a) concentrations: analysis of studies published from 1974-2000. Fertility and Sterility, 75, 898-915.

Goldberg, I.J.; L.-S. Huang; L.A. Huggins; S. Yu; P.R. Nagareddy; T.S. Scanlan \& J.R. Ehrenkranz (2012). Thyroid hormone reduces cholesterol via a non-LDL receptor-mediated pathway. Endocrinology, 153, 5143-5149.

Jia, L.; X. Liu; Y.Y. Bai; S.H. Li; K. Sun; C. He \& R. Hui (2010). Short-term effect of cocoa product consumption on lipid profile: a meta-analysis of randomized controlled trials. The American Journal of Clinical Nutrition, 92, 218-225.

Keen, C.L.; R.R. Holt; P.I. Oteiza; C.G. Fraga \& H.H. Schmitz (2005). Cocoa antioxidants and cardiovascular health. The American Journal of Clinical Nutrition, 81, 298S-303S.

Li, C.; M.R. Briggs; T.E. Ahlborn; F.B. Kraemer \& J. Liu (2001). Requirement of Sp1 and estrogen receptor $\alpha$ interaction in $17 \alpha$-estradiol-mediated transcriptional activation of the low density lipoprotein receptor gene expression. Endocrinology, 142, 1546-1553.
Michel, T.; M. Halabalaki \& A.-L. Skaltsounis (2013). New concepts, experimental approaches, and dereplication strategies for the discovery of novel phytoestrogens from natural sources. Planta Medica, 79, 514-532.

Mvondo, M.; D. Njamen; S.T. Fomum; J. Wandji \& G. Vollmer (2011). A postmenopause-like model of ovariectomized Wistar rats toidentify active principles of Erythrina lysistemon (Fabaceae). Fitoterapia, 82, 939-949.

Ohlsson, C.; N. Hellberg; P. Parini; O. Vidal; M. Bohlooly; M. Rudling; M.K. Lindberg; M. Warner; B. Angelin \& J.-Å. Gustafsson (2000). Obesity and disturbed lipoprotein profile in estrogen receptor- $\alpha$-deficient male mice. Biochemical and Biophysical Research Communications, 278, 640-645.

Saengsirisuwan, V.; S. Pongseeda; M. Prasannarong; K. Vichaiwong \& C. Toskulkao (2009). Modulation of insulin resistance in ovariectomized rats by endurance exercise training and estrogen replacement. Metabolism, 58, 38-47.

Smith, P.M.; A. Cowan \& B.A. White (2004). The low-density lipoprotein receptor is regulated by estrogen and forms a functional complex with the estrogenregulated protein ezrin in pituitary $\mathrm{GH} 3$ somatolactotropes. Endocrinology, 145, 3075-3083.

Tikkanen, M.; T. Kuusi; E. Vartiainen \& E. Nikkilä (1979). Treatment of post-menopausal hypercholerolaemia with estradiol. Acta Obstetricia et Gynecologica Scandinavica, 58, sup88, 83-88.

Urabe, M.; T. Yamamoto; T. Kashiwagi; T. Okubo; H. Tsuchiya; K. Iwasa; N. Kikuchi; K. Yokota; K. Hosokawa \& H. Honjo (1996). Effect of estrogen replacement therapy on hepatic triglyceride lipase, lipoprotein liase and lipids including apolipoprotein in climacteric and elderly women. Endocrine Journal, 43, 737-742.

Xu, B.; D. Lovre \& F. Mauvais-Jarvis (2016). Effect of selective estrogen receptor modulators on metabolic homeostasis. Biochimie, 124, 92-97.

$$
* * 0 * *
$$

